\title{
Effet du niveau et de la nature de l'azote dégradable sur la digestion ruminale d'un régime à base d'ensilage de maïs chez la vache laitière
}

\author{
JL Peyraud *, S Le Liboux, R Vérité \\ Station de recherches sur la vache laitière, Inra, 35590 St Gilles, France
}

(Reçu le 6 février 1996 ; accepté le 4 avril 1997)

\begin{abstract}
Summary - Effect of level and nature of the source of rumen degradable nitrogen on ruminal digestion in dairy cows fed with a maize silage based diet. Four diets differing in level and source of nitrogen were given in a $4 \times 4$ latin square design using four dairy cows canulated in the rumen and duodenum. A low $\mathrm{N}$ diet (T-, $17.4 \mathrm{~g} \mathrm{~N} / \mathrm{kg} \mathrm{DM}$ ) with only $17 \mathrm{~g}$ of degradable $\mathrm{N}$ per $\mathrm{kg}$ of fermentable OM (FOM) (covering $73 \%$ of microbial requirements) was supplemented with up to $21.6 \mathrm{~g} / \mathrm{kg} \mathrm{DM}$ and $22 \mathrm{~g} / \mathrm{kg}$ FOM (covering $95 \%$ of microbial requirements) of increasing levels of preformed amino acids. Three mixtures of urea and casein [100:0 (U); 50:50 (UC) and 0:100 (C)] were used in order to supply 30,15 and $0 \%$ of the total $N$ of the diet as urea-N. The four diets (66\% maize silage) were isoenergetic and were offered $95 \%$ of ad libitum twice daily. OM and NDF digestibilities in the total digestive tract and in the rumen did not differ between $\mathrm{T}$ - and $\mathrm{U}$ diets. These digestibilities increased linearly with the proportion of casein- $\mathrm{N}$ in the diet but the response remained low. The levels and the source of degradable $\mathrm{N}$ did not affect the non ammonia nitrogen (NNA) flow into the duodenum, the microbial $\mathrm{N}$ flow and the efficiency of microbial synthesis. NNA flow exceeded N intake for all diets but the net recycling into the rumen was markedly higher for the LN diet $(+110 \mathrm{vs}$ $+31 \mathrm{~g} /$ day). This is related to a lower excretion of urea- $\mathrm{N}$ in urine ( $22 \mathrm{vs} 80 \mathrm{~g} /$ day). These results suggest that in cows fed at $95 \%$ of ad libitum, the shortage of degradable $\mathrm{N}$ hardly affects the digestion of the diet and that the supply of preformed amino acids is not an important limiting factor for bacterial growth when maïze silage based diets are fed. Therefore, the supplementation with such preformed carbon chains is not of practical interest.
\end{abstract}

dairy cow / degradable nitrogen / rumen / microbial activity / duodenal flow

Résumé - Quatre régimes différents par le niveau et la source d'azote (N) dégradable ont été comparés dans un schéma en carré latin avec quatre vaches fistulées du rumen et du duodénum. Un régime témoin négatif ( $\mathrm{T}$-, $17,4 \mathrm{~g} \mathrm{~N} / \mathrm{kg} \mathrm{MS}$ ) ne couvrant que $73 \%$ des besoins en $\mathrm{N}$ dégradable des microbes (17 $\mathrm{g} \mathrm{N}$ dégradable/kg MO fermentescible) a été supplémenté jusqu’à $95 \%$ des besoins

\footnotetext{
* Correspondance et tirés à part
}

Tél : (33) 0299285100 ; fax : (33) 0299285101 ; courriel : peyraud@ st-gilles.rennes.inra.fr 
(21,6 g N/kg MS, $22 \mathrm{~g} \mathrm{~N} / \mathrm{kg}$ MO fermentescible) avec trois mélanges d'urée et de caséine [100:0 (U) ; $50: 50$ (UC) et 0:100 (C)], de manière à ce que l'azote uréique représente respectivement 30,15 et $0 \%$ de l'azote total du régime. Les quatre régimes (66\% d'ensilage de maïs) étaient a priori isoénergétiques et offerts en quantités légèrement limitées ( $95 \%$ de l'à volonté) en deux repas par jour. Les digestibilités totales et ruminales de MO et NDF n'ont pas été différentes entre les régimes T-et U. Elles ont augmenté linéairement avec la proportion d'azote caséinique, mais la réponse est restée modérée. Les flux d'azote non ammoniacal (NNA) et microbien et l'efficacité de la protéosynthèse ruminale n'ont pas été affectés par les traitements. Le flux de NNA a été supérieur aux quantités de N ingérées pour tous les régimes, mais le gain net a été sensiblement plus élevé pour le régime $T-(+110 \mathrm{vs}$ $+31 \mathrm{~g} / \mathrm{j}$ our) ce qui est à relier à la réduction de l'excrétion d'azote uréique dans l'urine observée avec ce régime. Ces résultats suggèrent que chez la vache laitière alimentée à $95 \%$ de l'ad libitum, une forte limitation de l'apport d'azote dégradable affecte modérément la digestion du régime et que la nature de l'azote dégradable des régimes à base d'ensilage de maïs ne constitue pas un facteur limitant important de l'activité microbienne. Dans ces conditions, la supplémentation avec des chaînes carbonées préformées n'a que peu d'intérêt pratique.

\section{vache laitière / azote dégradable / rumen / activité microbienne / flux duodénal}

\section{INTRODUCTION}

Les deux principales sources d'azote disponibles pour les microbes du rumen sont l'ammoniac et les acides aminés et peptides présents dans le milieu. Bien que la plupart des bactéries du rumen puissent se développer dans des milieux contenant exclusivement de l'ammoniac comme source d'azote, le besoin en chaînes aminées préformées pour maximiser la croissance microbienne a été clairement identifié avec des études in vitro (Maeng et al, 1976; Cotta et Russel, 1982 ; Argyle et Baldwin, 1989). Les peptides apparaissent avoir un effet stimulant parfois plus marqué que celui des acides aminés libres (Wright, 1967 ; Argyle et Baldwin, 1989), certaines espèces comme Bactérö̈des Ruminicola ayant même des besoins spécifiques en peptides (Pittman et Bryant, 1964). De même, lorsque l'azote uréique représente $90 \%$ de l'azote total de régimes purifiés distribués à des ruminants, l'efficacité des synthèses microbiennes est accrue par la substitution d'une partie de l'urée par des acides aminés ou des peptides (Hume, 1970 ; Leibholz et Kellaway, 1979).

Dans des situations plus proches des pratiques classiques d'alimentation, l'urée représente bien moins de $40 \%$ de l'azote dégradable du régime et l'effet de la sub- stitution d'azote non protéique par des protéines dégradables sur la protéosynthèse est alors faible (Amos et Evans, 1976 ; Cecava et al, 1988) ou nul (Chen et al, 1992 ; Kropp et al, 1977 ; Cecava et Parker, 1993), sans doute parce que les besoins en acides aminés et peptides préformés des bactéries sont alors partiellement couverts par les protéines dégradables du régime.

Cependant, pour des vaches laitières à bon niveau de production, la protéosynthèse doit être maximisée et la nature de l'azote dégradable pourrait alors avoir un intérêt pratique, notamment dans le cas de régimes moins riches en protéines et utilisant une proportion importante de protéines peu dégradables pour limiter les rejets azotés. Cet essai vise à déterminer l'effet du niveau et de la nature de l'azote dégradable sur la digestion de l'énergie et des protéines chez des vaches laitières recevant un régime à base d'ensilage de maïs.

\section{MATÉRIELS ET MÉTHODES}

\section{Traitements, schéma expérimental et animaux}

Quatre régimes différant par le niveau et la nature de l'azote dégradable ont été comparés dans un 
schéma en carré latin $4 \times 4$. Un régime témoin négatif ( $\mathrm{T}-, 17,4 \mathrm{~g} \mathrm{~N} / \mathrm{kg} \mathrm{MS}$ ) ne couvrant a priori que $73 \%$ des besoins des microbes en $\mathrm{N}$ dégradable et ne contenant pas d'urée a été complémenté de manière à couvrir $95 \%$ des besoins des microbes soit par de l'urée seule (U), soit par un mélange 50/50 d'urée et de caséine (UC) soit par de la caséine seule (C). Les trois régimes supplémentés comportaient $21,6 \mathrm{~g} \mathrm{~N} / \mathrm{kg} \mathrm{MS}$. Les besoins en $\mathbf{N}$ dégradable des microbes établis selon les recommandations du système PDI sont de $23,2 \mathrm{~g} / \mathrm{kg}$ MO fermentescible (MOF) (Vérité et Peyraud, 1989). Le régime $\mathrm{T}$ - et les régimes supplémentés apportaient donc 17 et $22 \mathrm{~g} \mathrm{~N}$ dégradable/kg MOF respectivement. L'azote uréique représentait 30,15 et $0 \%$ de l'azote dégradable pour les régimes $\mathrm{U}, \mathrm{UC}$ et $\mathrm{C}$ respectivement (tableau I).

L'essai a été conduit avec quatre vaches laitières multipares équipées d'une large canule du rumen (diamètre intérieur $12 \mathrm{~cm}$ ) et d'une canule en T située dans le duodénum proximal. Chaque période expérimentale a duré 4 semaines.

\section{Constitution des régimes et alimentation}

Les régimes ont été constitués principalement par de l'ensilage de maïs et un concentré énergétique à base de céréales (tableau I). Afin de contenir essentiellement des protéines peu dégradables, le régime témoin négatif a été complémenté par du tourteau de soja-colza tanné. Un apport fixe de $500 \mathrm{~g}$ de tourteau de soja non tanné a toutefois été maintenu, afin de limiter à moins de $250 \mathrm{~g}$ par vache et par jour les quantités d'urée à apporter dans le régime U. Pour les régimes $\mathrm{U}$, UC et $\mathrm{C}$, un mélange d'amidon de maïs, d'urée et de caséine a été substitué de manière iso-énergétique à $4 \%$ du concentré à base de céréales. Les quantités de caséine (sous forme de caséinate de calcium), d'urée et d'amidon de blé à apporter ont été calculées à partir de leur teneur en PDIN et en énergie nette sur la base de : 1 caséine $=0,328 \times$ urée $+1 \times$ amidon de blé. La valeur PDIN de la caséine a été estimée en supposant une dégradation totale dans le rumen et un taux de captation de l'azote dégradé égal à celui de l'urée (0,8; Vérité et Peyaud, 1989). La

Tableau I. Composition et valeur nutritive des régimes.

\begin{tabular}{|c|c|c|c|c|}
\hline & $T-$ & $U$ & $U C$ & $C$ \\
\hline \multicolumn{5}{|l|}{ Composition des régimes (\% MS) } \\
\hline Ensilage de maïs & 66,5 & 66,5 & 66,5 & 66,5 \\
\hline Tourteau de soja ${ }^{a}$ & 5,5 & 5,5 & 5,5 & 5,5 \\
\hline Minéraux et vitamines & 1,5 & 1,5 & 1,5 & 1,5 \\
\hline Concentré énergie ${ }^{b}$ & 26,5 & 22,5 & 22,5 & 22,5 \\
\hline Urée & 0,0 & 1,0 & 0,5 & 0,0 \\
\hline Caséine $^{\mathrm{c}}$ & 0,0 & 0,0 & 1,6 & 3,2 \\
\hline Amidon de blé & 0,0 & 3,2 & 1,6 & 0,0 \\
\hline \multicolumn{5}{|l|}{ Valeur nutritive attendue (/kg MS) } \\
\hline Énergie nette (UFL) & 0,93 & 0,92 & 0,93 & 0,93 \\
\hline g MAT & 109 & 135 & 135 & 136 \\
\hline $\mathrm{g}$ PDIE & 90 & 88 & 89 & 90 \\
\hline g PDIN $^{d}$ & 73 & 86 & 85 & 87 \\
\hline dont $\mathrm{N}$ urée ( $\% \mathrm{~N}$ dégradable) ${ }^{\mathrm{d}}$ & 0 & 30 & 15 & 0 \\
\hline
\end{tabular}

T- : régime témoin négatif ne couvrant que $73 \%$ des besoins des microbes en $\mathrm{N}$ dégradable ; $\mathrm{U}, \mathrm{UC}$ et $\mathrm{C}$ : régimes supplémentés de manière à couvrir $95 \%$ des besoins des microbes par de l'urée seule (U), un mélange 50:50 d'urée et de caséine (UC) ou de la caséine seule (C) ; ${ }^{a}$ dont $50 \%$ de tourteau tanné ; ${ }^{b}$ sur la base de la MS (\%) : 35 blé, 35 maïs, 23 orge, 3 mélasse, $3 \mathrm{CMV}, 1$ matières grasses ; ${ }^{\mathrm{C}}$ caséinate de calcium ; ${ }^{\mathrm{d}} \mathrm{La}$ valeur azotée des régimes a été calculée selon le système PDI (Vérité et Peyraud, 1989). 
composition des aliments est donnée au tableau II.

Le plan de rationnement a été établi de manière à couvrir les besoins énergétiques et seulement $95 \%$ des besoins azotés attendus des animaux, pour limiter les quantités d'azote pouvant être recyclées. Les rations ont été distribuées en quantités légèrement limitées $(95 \%$ de l'ad libitum) en deux repas égaux à $7 \mathrm{~h} 00$ et 17h30. Le mélange urée-amidon de blé, la caséine et les concentrés de production ont été intimement mélangés au fourrage dans l'auge. Tous les régimes ont été supplémentés par $300 \mathrm{~g}$ de complément minéral vitaminique et les animaux ont eu libre accès pendant tout l'essai à une pierre à lécher.

\section{Procédure expérimentale}

Chaque période de 4 semaines a consisté en 2 semaines d'adaptation aux régimes expérimentaux et 2 semaines de mesures. Les fèces et l'urine ont été collectées séparément et en totalité de $\mathrm{j} 18$ à $\mathrm{j} 24$. Les échantillons de contenus duodénaux et de rumen ont été prélevés de j18 à j24. La dynamique postprandiale des teneurs en ammoniac $\left(\mathrm{NH}_{3}\right)$ du contenu de rumen a été suivie par des séries de prélèvements à $\mathrm{j} 17$. Le taux de renouvellement des liquides du rumen a été mesuré à $\mathrm{j} 15$ et $\mathrm{j} 16$. Une prise de sang a été effectuée le matin et le soir de j22 pour la détermination de l'urémie. La production de lait a été mesurée de j15 à j27 et les taux ont été déterminés sur six traites consécutives de $\mathrm{j} 16$ à $\mathrm{j} 18$ puis de $\mathrm{j} 23$ à j25.

Pour la mesure de la digestibilité, les quantités brutes de fèces émises ont été pesées chaque jour. Une aliquote de $1 \%$ des fèces a été séchée en étuve ( 72 heures à $\left.80^{\circ} \mathrm{C}\right)$ pour la détermination de la matière sèche, puis ces échantillons journaliers ont été regroupés par vache et par période pour le dosage ultérieur de l'ytterbium. Une seconde aliquote de $0,5 \%$ a été congelée à $-20^{\circ} \mathrm{C}$, puis lyophilisée et broyée pour les analyses de composition chimique. Une dernière aliquote de $0,5 \%$ a été conservée à $4{ }^{\circ} \mathrm{C}$ pour le dosage du polyéthylène glycol (PEG). L'urine a été collectée pendant les 7 jours de digestibilité via un sac de plastique de $5 \mathrm{~L}$ attaché à un harnais fixé sur les animaux et pompée automatiquement après chaque miction dans un récipient contenant $500 \mathrm{~mL}$ de conservateur $\left(\mathrm{H}_{2} \mathrm{SO}_{4}\right.$ à $20 \%$ ). Une aliquote journalière de $0,5 \%$ a permis de constituer un échantillon moyen sur la période de collecte. Elle a été conservée à $-20^{\circ} \mathrm{C}$ jusqu'à l'analyse de l'azote total et de l'azote uréique.

Les flux des nutriments au duodénum ont été obtenus à partir d'une perfusion intra-ruminale continue de PEG (250 g/jour) et de chlorure d'ytterbium (2 g/jour d'ytterbium) (Mambrini et Peyraud, 1994). La perfusion a démarré à j10 à 9 h00, après une surcharge initiale ( $250 \mathrm{~g}$ de $\mathrm{PEG}$ et $2 \mathrm{~g}$ d'ytterbium), et a été maintenue jusqu'à j25 au moyen de deux pompes péristaltiques multicanaux avec un débit de respectivement 200 et $42 \mathrm{~mL} /$ heure pour le PEG et l'ytterbium. Vingtquatre prélèvements de contenu duodénal $(300 \mathrm{~mL})$ ont été réalisés à intervalle de 1 heure pour couvrir le nycthémère. Trois échantillons moyens ont été constitués. Une aliquote de $40 \mathrm{~mL}$ a été conservée à $4{ }^{\circ} \mathrm{C}$ pour l'analyse du PEG. Une seconde aliquote de $80 \mathrm{~mL}$ a été conservée à $4{ }^{\circ} \mathrm{C}$ pour l'isolation des bactéries. Le reste du prélèvement a été conservé à $-20^{\circ} \mathrm{C}$ avant d'être lyophilisé pour les analyses ultérieures.

Tableau II. Composition chimique (g/kg MS) des aliments.

\begin{tabular}{lccccccc}
\hline & $\begin{array}{c}\text { Ensilage } \\
\text { maïs }\end{array}$ & $\begin{array}{c}\text { Concentré } \\
\text { énergie }\end{array}$ & $\begin{array}{c}\text { Tourteau } \\
\text { soja }\end{array}$ & $\begin{array}{c}\text { Tourteau } \\
\text { tanné }\end{array}$ & Urée & $\begin{array}{c}\text { Caséine } \\
\text { Amidon } \\
\text { blé }\end{array}$ \\
\hline MO & 956 & 950 & 932 & 925 & 980 & 980 & 998 \\
MAT & 78 & 117 & 471 & 476 & 2875 & 937 & 6 \\
NDF & 438 & 165 & 164 & 243 & 0 & 10 & 2 \\
ADF & 246 & 43 & 86 & 113 & 0 & 8 & 1 \\
ADL & 25 & 9 & 7 & 21 & 0 & 0 & 0 \\
Amidon & 287 & 593 & 84 & 43 & 0 & 0 & 990 \\
\hline
\end{tabular}


Le flux microbien a été estimé par référence à la teneur en acide diamino pimélique (DAP) de l'échantillon de bactéries isolées des contenus de duodénum. Le contenu du duodénum a été centrifugé à $1000 \mathrm{~g}$ pendant $15 \mathrm{~min}$ (Jouan $\mathrm{K} 110$, rotor de $6 \times 11$ ). Le surnageant a été récupéré en totalité, puis ultracentrifugé ( $30000 \mathrm{~g}$ pendant $15 \mathrm{~min}$ ) avec une centrifugeuse à flux continu (CEPA Carl Padberg Type LE) (soit environ trois passages du surnageant avec un débit de $0,4 \mathrm{~L} / \mathrm{min}$ ). Le résidu a été rincé par passage dans la centrifugeuse de sérum physiologique (environ $2 \mathrm{~L}$ avec le même débit). Le résidu final a été récupéré, lyophilisé et conservé jusqu' aux analyses.

Pour la détermination du $\mathrm{pH}$ et des concentrations moyennes en $\mathrm{NH}_{3}$ et $\mathrm{AGV}$, le contenu du rumen a été prélevé dans le sac ventral aux mêmes heures que le contenu du duodénum. A chaque point de prélèvement, $50 \mathrm{~mL}$ de contenu du sac ventral ont été aspirés à la canne. Le $\mathrm{pH}$ a été immédiatement mesuré, puis le contenu a été filtré sur six épaisseurs de mousseline. Une aliquote de $4 \mathrm{~mL}$ a été diluée avec $4 \mathrm{~mL}$ de conservateur $(\mathrm{NaCl}$ à $20 \%)$ et conservée à $-20^{\circ} \mathrm{C}$ pour l'analyse de $\mathrm{NH}_{3}$. Un échantillon de $4 \mathrm{~mL}$ a été mélangé avec $0,4 \mathrm{~mL}$ de conservateur $\left(\mathrm{H}_{3} \mathrm{PO}_{4}\right.$ à $5 \%$ et $\mathrm{HgCL}_{2}$ à $1 \%$ ) et stocké à $-20^{\circ} \mathrm{C}$ pour l'analyse des AGV.

La cinétique postprandiale de l'ammoniac a été suivie par des prélèvements de contenu ruminal à $7 \mathrm{~h}$ (avant la distribution du repas), 8, 9, $10,11,13,15$ et $17 \mathrm{~h}$. Ces échantillons ont été traités de la même manière que les échantillons moyens. Le taux de renouvellement des liquides du rumen a été mesuré par la décroissance sur 24 heures dans le rumen d'une surcharge de chrome EDTA ( $11 / \mathrm{vache} ; 1,5 \mathrm{~g} \mathrm{Cr} / \mathrm{L})$, introduite à $11 \mathrm{~h} 00$. Des échantillons de contenu de rumen ont été prélevés par aspiration à $15 \mathrm{~h}$, $15 \mathrm{~h} 30$ et $16 \mathrm{~h}$ le jour même et le lendemain. Ils ont été filtrés sur six épaisseurs de mousseline et conservés à $-20^{\circ} \mathrm{C}$ jusqu'au dosage du $\mathrm{Cr}$ EDTA.

\section{Analyses chimiques}

Les analyses (MO, N, constituants pariétaux) ont été effectuées sur les échantillons d'aliments, de feces et de contenus duodénaux après broyage à travers une grille de $0,8 \mathrm{~mm}$. La teneur en $\mathrm{MO}$ a été déterminée après calcination des échantillons secs à $550^{\circ} \mathrm{C}$ pendant 6 heures. L'azote total a été déterminé par la méthode de Kjeldahl, la distillation et le titrage de $\mathrm{NH}_{3}$ étant réalisés automatiquement sur un appareil Buchi 322. Les constituants pariétaux (NDF) ont été dosés en séquence sur Fibertec, selon la méthode proposée initialement par Van Soest (1963) et redéfinie par Giger et Pochet (1987). L'azote ammoniacal a été dosé selon la méthode de Berthelot adaptée à l'auto-analyseur Technicon. L'urée du sang a été dosée par colorimétrie sur l'auto-analyseur Technicon, selon la méthode préconisée par Michel (1971). Les AGV ont été déterminés par chromatographie en phase gazeuse (Varian 3400 , Varian France, SA) avec une colonne remplie de $1,5 \mathrm{~m}$ de longueur et $2 \mathrm{~mm}$ de diamètre interne et de l'azote comme gaz vecteur (Jouany, 1982). Le PEG a été dosé par la méthode turbidimétrique de Hyden (1955), modifiée par Malawer et Powell (1967). L'ytterbium a été déterminé par spectrophotométrie d'absorption atomique, après minéralisation à sec des échantillons et reprise des cendres à chaud ( 3 min d'ébullition sur une plaque chauffante) par une solution d'acide nitrique $1,5 \mathrm{~N}$ et contenant $2 \%$ de chlorure de potassium (Mambrini et Peyraud, 1994). Les gammes ont été préparées à partir des contenus digestifs et de fèces prélevés avant la perfusion des marqueurs. Le Cr EDTA a été déterminé au spectrophotomètre d'absorption atomique, après centrifugation des échantillons de contenu ruminal mais sans minéralisation préalable. Le DAP a été analysé par chromatographie en phase liquide sur un auto-analyseur Biotronik LL 5001 selon la méthode décrite par Le Hénaff (1991). Brièvement, $200 \mathrm{mg}$ d'échantillon de bactéries et de contenus duodénaux ont été hydrolysés pendant 24 heures dans de l'acide $(\mathrm{HCl} 6 \mathrm{~N})$ sous reflux atmosphérique et dans un bain d'huile thermostatée à $110^{\circ} \mathrm{C}$, après addition de $2,5 \mathrm{mg}$ de norleucine comme marqueur interne.

\section{Calculs}

Les flux de nutriments au duodénum ont été calculés par la méthode du simple marquage, en divisant la quantité de marqueurs récupérée dans les fèces par les teneurs moyennes en ytterbium et PEG du contenu duodénal prélevé. La moyenne des deux estimations a été retenue (Mambrini et Peyraud, 1994). La proportion d'azote microbien dans le flux duodénal a été calculée en divisant la valeur du rapport DAP/N du contenu du duodénum par la valeur du rapport DAP/N de l'échantillon de bactéries de référence. 


\section{Analyses statistiques}

Les données moyennes de production, de flux digestifs, de paramètres de la digestion ruminale et d'urémie ont été étudiées par analyse de variance, selon un schéma en carré latin avec la procédure des modèles linéaires généraux (GLM, Statistical Analysis System, 1987). La variabilité totale a été séparée en un effet des traitements ( $3 \mathrm{ddl})$, des animaux ( $3 \mathrm{ddl}$ ) et des périodes ( $3 \mathrm{ddl})$ et une résiduelle ( $6 \mathrm{ddl})$. Trois contrastes orthogonaux indépendants ont été retenus pour tester l'effet du niveau d'azote dégradable du régime ( $\mathrm{T}$ - vs $(\mathrm{U}+\mathrm{UC}+\mathrm{C}) / 3$; Niv N) et analyser les courbes de réponse à l'apport de proportions croissantes de caséine dans l'azote dégradable du régime (linéaire + quadratique ; Lin, Quad), selon la méthode des polygones orthogonaux. Aucun effet rémanent des traitements n'a été mis en évidence.

Les données des cinétiques postprandiales ont été analysées selon un schéma en split plot intégrant une double résiduelle selon le modèle
$\mathrm{Y}_{\mathrm{ijkl}}=\mathrm{Moy}+\mathrm{A}_{\mathrm{i}}+\mathrm{P}_{\mathrm{j}}+\mathrm{T}_{\mathrm{k}}+\mathrm{E}_{\mathrm{ijk}}+\mathrm{H}_{1}+(\mathrm{AH})_{\mathrm{il}}$ $+(\mathrm{PH})_{\mathrm{jl}}+(\mathrm{TH})_{\mathrm{kl}}+\mathrm{E}_{\mathrm{j} \mathrm{jk} !}$ où $\mathrm{A}, \mathrm{P}, \mathrm{T}, \mathrm{H}$ représentent les effets des animaux, des périodes, des traitements ( $3 \mathrm{ddl}$ ) et de l'heure ( $7 \mathrm{ddl}$ ) et $\mathrm{AH}$, $\mathrm{PH}, \mathrm{TH}$ les effets des interactions de l'heure avec les animaux, les périodes et les traitements (21 ddl chacune). Les mêmes contrastes orthogonaux ont été utilisés.

\section{RÉSULTATS}

Les quantités de MO ingérées ont été de $18,7 \mathrm{~kg}$ en moyenne. Comparée au régime $\mathrm{T}$-, l'augmentation des apports d'azote dégradable a accru la digestibilité totale et ruminale de la MO ainsi que la proportion de la MO digestible apparemment dégradée dans le rumen (tableau III). En conséquence, les quantités ingérées de MO digestible (MOD) et de MO apparemment fermentée

Tableau III. Effet du niveau et de la nature des matières azotées dégradables sur la digestion de la matière organique, des constituants pariétaux et de l'amidon.

Contrastes

$T-\quad U \quad U C \quad C \quad E^{a}$ NivN Lin Quad

\begin{tabular}{|c|c|c|c|c|c|c|c|c|}
\hline \multicolumn{9}{|l|}{ MO } \\
\hline ingérée (kg/jour) & 18,54 & 18,84 & 18,91 & 18,62 & 0,55 & $\mathrm{~ns}$ & $\mathrm{~ns}$ & ns \\
\hline digestibilité totale & 0,684 & 0,701 & 0,708 & 0,716 & 0,0127 & 0,01 & 0,10 & ns \\
\hline dont rumen $(\%)$ & 56,4 & 57,0 & 64,4 & 60,5 & 3,04 & 0,03 & 0,11 & 0,04 \\
\hline $\begin{array}{l}\text { digestibilité apparente } \\
\text { rumen }\end{array}$ & 0,386 & 0,400 & 0,457 & 0,434 & 0,0256 & 0,03 & 0,10 & 0,04 \\
\hline \multicolumn{9}{|l|}{ NDF } \\
\hline ingéré ( $\mathrm{kg} /$ jour $)$ & 6,80 & 6,80 & 6,77 & 6,64 & 0,255 & ns & ns & ns \\
\hline digestibilité totale & 0,425 & 0,434 & 0,450 & 0,462 & 0,0143 & 0,03 & 0,03 & ns \\
\hline digestibilité ruminale & 0,357 & 0,385 & 0,429 & 0,427 & 0,0441 & 0,07 & 0,14 & ns \\
\hline \multicolumn{9}{|l|}{ Amidon } \\
\hline ingéré ( $\mathrm{kg} / \mathrm{j}$ our) & 6,98 & 7,17 & 7,02 & 6,68 & 0,224 & ns & 0,02 & ns \\
\hline digestibilité totale & 0,942 & 0,949 & 0,951 & 0,951 & 0,0122 & ns & ns & ns \\
\hline digestibilité ruminale & 0,705 & 0,695 & 0,769 & 0,726 & 0,0243 & 0,12 & 0,12 & 0,01 \\
\hline
\end{tabular}

T- : régime témoin négatif couvrant $73 \%$ des besoins des microbes en $\mathrm{N}$ dégradable ; $\mathrm{U}, \mathrm{UC}$ et $\mathrm{C}$ : régimes supplémentés de manière à couvrir $95 \%$ des besoins des microbes par de l'urée seule (U), un mélange 50:50 d'urée et de caséine (UC) ou de la caséine seule (C) ; ${ }^{\text {a }}$ écart-type résiduel de l'analyse de variance. 
dans le rumen ont été accrues de respectivement 0,64 et $0,95 \mathrm{~kg} / \mathrm{jour}(p<0,05)$. La digestibilité totale de la $\mathrm{MO}$ a eu tendance à augmenter linéairement avec la proportion de $\mathrm{N}$ caséinique $(p<0,10)$. La proportion de la MO digestible dégradée dans le rumen et la digestibilité ruminale de la MO n'ont pas été différentes entre les régimes $\mathrm{T}$ - et $\mathrm{U}$ mais ont été accrues avec les régimes UC et $\mathrm{C}$ (tableau III). Ainsi, les quantités de MO apparemment fermentées dans le rumen se sont accrues de plus de $0,6 \mathrm{~kg} /$ jour avec les régimes UC et $\mathrm{C}(p<0,05)$.

L'augmentation de l'apport d'azote dégradable a entraîné une augmentation modérée de la digestibilité totale des constituants pariétaux $(+0,02$ unité, $p<0,03)$ et de leur digestibilité ruminale $(+0,06$ unité, $p<0,07$ ) (tableau III). La digestibilité totale et ruminale des constituants pariétaux a été peu différente entre les régimes $\mathrm{T}$ - et $\mathrm{U}$ et a eu tendance à s'accroître linéairement avec la proportion d' $N$ caséinique. La digestibilité totale de l'amidon n'a pas été affectée par les traitements. Sa digestibilité dans le rumen a été semblable entre les régimes $T$ et $\mathrm{U}$ et a évolué de manière quadratique avec la proportion d' $\mathrm{N}$ caséinique, le régime UC conduisant aux valeurs les plus élevées.

Le flux d'azote non ammoniacal entrant dans l'intestin (NNA), exprimé en g/jour ou rapporté à la $\mathrm{MO}$ digestible ingérée (460 g/jour ; $35,0 \mathrm{~g} / \mathrm{kg}$ MOD), les flux d'azote d'origine microbienne ( $277 \mathrm{~g} / \mathrm{jour})$ et d'origine alimentaire ( $154 \mathrm{~g} /$ jour $) \mathrm{n}$ 'ont pas été affectés significativement par le niveau et la nature de l'azote dégradable du régime (tableau IV). De même, les traitements n'ont pas modifié l'efficacité des synthèses microbiennes et la digestibilité apparente du NNA. Le flux de NNA a toujours été supérieur aux quantités d' $\mathrm{N}$ ingéré, mais la différence a été beaucoup plus faible avec

Tableau IV. Effet du niveau et de la nature des matières azotées dégradables sur la digestion de l'azote.

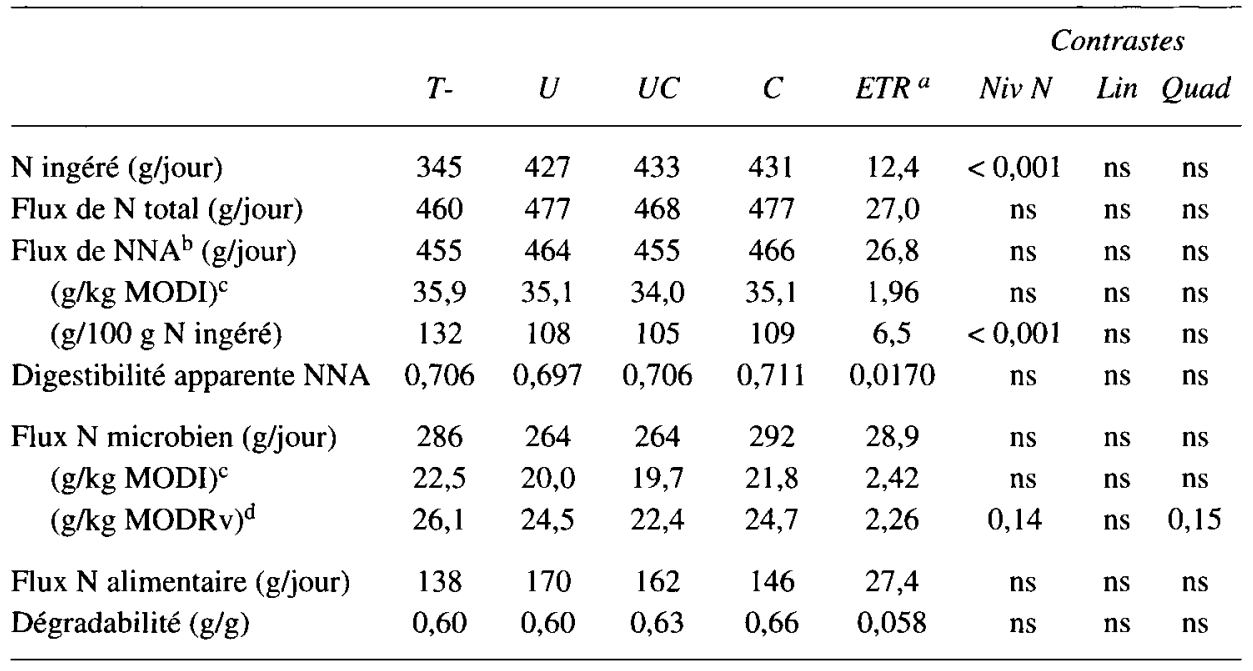

T- : régime témoin négatif couvrant $73 \%$ des besoins des microbes en $\mathrm{N}$ dégradable ; $\mathrm{U}, \mathrm{UC}$ et $\mathrm{C}:$ régimes supplémentés de manière à couvrir $95 \%$ des besoins des microbes par de l'urée seule (U), un mélange 50:50 d'urée et de caséine (UC) ou de la caséine seule (C); ${ }^{a}$ écart-type résiduel de l'analyse de variance ; ${ }^{b} \mathrm{~N}$ non ammoniacal ; ${ }^{c}$ MO digestible ingérée ; ${ }^{d}$ MO réellement dégradée dans le rumen = MOI - flux MO + flux MO microbienne. 
les trois régimes supplémentés qu'avec le régime $\mathrm{T}-(p<0,001)$.

L'excrétion d'azote dans les fèces n'a pas été affectée par les traitements. L'augmentation des apports d'azote dégradable a entraîné une forte augmentation de l'excrétion d'azote total et d'azote uréique dans l'urine ainsi que de l'urémie $(p<0,001$; tableau V). En revanche, la nature de l'azote dégradable n'a pas affecté l'excrétion d'N urinaire et l'urémie. La quantité d'N non uréique excrétée dans l'urine n'a pas varié entre les traitements ( $43 \mathrm{~g} /$ jour en moyenne).

La teneur en $\mathrm{NH}_{3}$ du contenu ruminal a été beaucoup plus élevée avec les régimes enrichis en $\mathrm{N}$ dégradable $(p<0,001$; tableau VI), cette différence étant maintenue pratiquement tout au long de la journée (fig 1). La teneur en $\mathrm{NH}_{3}$ a diminué de manière linéaire avec la teneur en $\mathrm{N}$ caséinique du régime. En particulier, le pic de $\mathrm{NH}_{3}$ après la distribution des repas a été décalé et moins marqué pour le régime $\mathrm{C}$ que pour les régimes contenant de l'urée (fig 1). Le niveau et la nature de l'azote dégradable n'ont eu d'effet ni sur le $\mathrm{pH}(6,35$ en moyenne) ni sur l'acidité totale du rumen
( $98 \mathrm{mmol} / \mathrm{L}$ en moyenne). La proportion d'AGV ramifiés (AGVR) n'a pas été différente entre les régimes $T$ - et U mais s'est accrue linéairement avec la proportion d'N caséinique (tableau VI). La proportion d'acide acétique a évolué en sens inverse de celle des AGVR. Le taux de renouvellement des liquides du rumen n'a pas été affecté par les traitements (tableau VI).

L'augmentation des apports d'azote dégradable a entraîné une augmentation de la production de lait et de matières mais les différences n'ont été significatives que pour la production de lait $(+1,4 \mathrm{~kg}, p<0,10)$ (tableau VII). Les taux butyreux et protéique n'ont pas été affectés. L'accroissement de la proportion de $\mathrm{N}$ caséinique n'a affecté ni la production ni les taux (tableau VII).

\section{DISCUSSION}

\section{Fourniture de nutriments azotés pour les microbes}

Avec le régime $\mathrm{T}$-, la teneur en $\mathrm{NH}_{3}$ du contenu du rumen a été en moyenne de $51 \mathrm{mg} / \mathrm{L}$ et des concentrations proches de

Tableau V. Effet du niveau et de la nature des matières azotées dégradables sur le bilan azoté des animaux et l'urémie.

\begin{tabular}{lccccccccc}
\hline & $T-$ & $U$ & $U C$ & $C$ & $E T R^{a}$ & Niv N & Lin Quad \\
& & & & & & & & \\
Bilan N (g/jour) & & & & & & & & \\
$\quad$ N ingéré & 135 & 427 & 433 & 431 & 12,4 & $\mathrm{~ns}$ & $\mathrm{~ns}$ & $\mathrm{~ns}$ \\
$\quad$ N fèces & 133 & 140 & 133 & 133 & 4,8 & $\mathrm{~ns}$ & $\mathrm{~ns}$ & $\mathrm{~ns}$ \\
$\quad$ N urine & 61 & 126 & 124 & 122 & 8,5 & $<0,001$ & $\mathrm{~ns}$ & $\mathrm{~ns}$ \\
$\quad$ dont N uréique & 22 & 83 & 83 & 75 & 7,1 & $<0,001$ & $\mathrm{~ns}$ & $\mathrm{~ns}$ \\
$\quad$ N lait & 134 & 135 & 138 & 142 & 5,7 & $\mathrm{~ns}$ & 0,11 & $\mathrm{~ns}$ \\
Urémie (mg/100 $\mathrm{mL})$ & 7,7 & 19,1 & 20,6 & 21,4 & 1,93 & $<0,01$ & $\mathrm{~ns}$ & $\mathrm{~ns}$ \\
\hline
\end{tabular}

T- : régime témoin négatif couvrant $73 \%$ des besoins des microbes en $\mathrm{N}$ dégradable ; $\mathrm{U}$, $\mathrm{UC}$ et $\mathrm{C}:$ régimes supplémentés de manière à couvrir $95 \%$ des besoins des microbes par de l'urée seule (U), un mélange 50:50 d'urée et de caséine (UC) ou de la caséine seule (C). a écart-type résiduel de l'analyse de variance. 
Tableau VI. Effet du niveau et de la nature des matières azotées dégradables sur le $\mathrm{pH}$, les produits terminaux de la digestion ruminale (données moyennes sur le nycthémère) et le taux de renouvellement des liquides.

\begin{tabular}{|c|c|c|c|c|c|c|c|c|}
\hline & \multirow[b]{2}{*}{$T$} & \multirow[b]{2}{*}{$U$} & \multirow[b]{2}{*}{$U C$} & \multirow[b]{2}{*}{$C$} & \multirow[b]{2}{*}{$E_{T R}^{a}$} & \multicolumn{3}{|c|}{ Contrastes } \\
\hline & & & & & & $\operatorname{Niv} N$ & $\operatorname{Lin}$ & Quad \\
\hline Ammoniac $(\mathrm{mg} / \mathrm{L})$ & 51 & 149 & 138 & 128 & 8,6 & $<0,001$ & 0,01 & ns \\
\hline $\mathrm{pH}$ & 6,35 & 6,38 & 6,35 & 6,33 & 0,075 & ns & ns & ns \\
\hline Acidité totale (mmoles/L) & 95 & 98 & 99 & 99 & 3,8 & ns & ns & ns \\
\hline Ac acétique $(\%)$ & 62,7 & 63,3 & 62,2 & 61,5 & 0,78 & ns & 0,02 & ns \\
\hline Ac propionique (\%) & 18,1 & 17,7 & 17,9 & 18,2 & 0,73 & ns & ns & ns \\
\hline Ac butyrique (\%) & 14,4 & 14,4 & 15,0 & 14,5 & 0,66 & ns & ns & ns \\
\hline Ac ramifiés $(\%)^{b}$ & 2,8 & 2,7 & 2,9 & 3,5 & 0,20 & 0,05 & $<0,001$ & $1 \mathrm{~ns}$ \\
\hline Ac mineurs $(\%)^{\mathrm{c}}$ & 2,1 & 2,0 & 2,1 & 2,2 & 0,21 & ns & $\mathrm{ns}$ & $\mathrm{ns}$ \\
\hline $\mathrm{K}$ liquides $(\% / \mathrm{h})^{\mathrm{d}}$ & 11,2 & 11,0 & 10,3 & 10,9 & 0,93 & ns & ns & ns \\
\hline
\end{tabular}

T- : régime témoin négatif couvrant $73 \%$ des besoins des microbes en $\mathrm{N}$ dégradable ; $\mathrm{U}$, UC et $\mathrm{C}:$ régimes supplémentés de manière à couvrir $95 \%$ des besoins des microbes par de l'urée seule (U), un mélange 50:50 d'urée et de caséine (UC) ou de la caséine seule (C) ; ${ }^{a}$ écart type résiduel de l'analyse de variance ; ${ }^{b}$ somme des acides iso-butyrique et iso-valérique; c somme des acides valérique et caproïque ; ${ }^{d}$ taux de renouvellement des liquides du rumen.

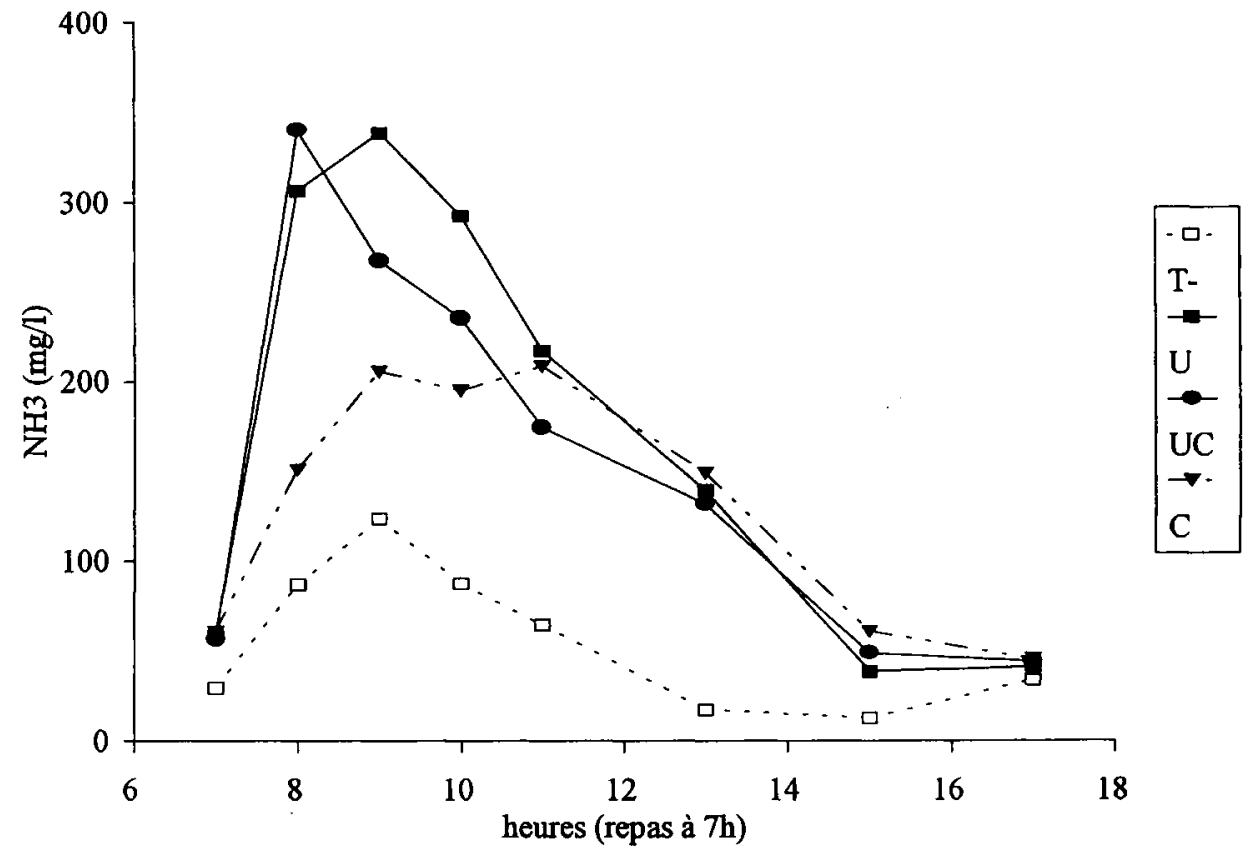

Fig 1. Evolution postprandiale de la teneur en ammoniac du contenu de rumen.

T- : régime témoin négatif couvrant $73 \%$ des besoins des microbes en $\mathrm{N}$ dégradable ; $\mathrm{U}, \mathrm{UC}$ et $\mathrm{C}$ : régimes supplémentés de manière à couvrir $95 \%$ des besoins des microbes par de l'urée seule (U), un mélange 50:50 d'urée et de caséine (UC) ou de la caséine seule (C). 
Tableau VII. Effet du niveau et de la nature des matières azotées dégradables sur la production et la composition du lait.

\begin{tabular}{lccccccccc}
\hline & & & & & \multicolumn{4}{c}{ Contrastes } \\
& $T-$ & $U$ & $U C$ & $C$ & $E^{a}$ & Niv N & Lin & Quad \\
\hline Lait brut (kg/jour) & 26,6 & 27,3 & 28,4 & 28,4 & 1,13 & 0,07 & $\mathrm{~ns}$ & $\mathrm{~ns}$ \\
Taux butyreux (g/kg) & 45,4 & 45,7 & 43,1 & 44,5 & 1,78 & $\mathrm{~ns}$ & $\mathrm{~ns}$ & 0,10 \\
Taux protéique (g/kg) & 30,9 & 30,9 & 29,7 & 30,0 & 1,40 & $\mathrm{~ns}$ & $\mathrm{~ns}$ & $\mathrm{~ns}$ \\
Matières grasses (g/jour) & 1182 & 1232 & 1193 & 1230 & 39,6 & $\mathrm{~ns}$ & $\mathrm{~ns}$ & $\mathrm{~ns}$ \\
Matières protéiques (g/jour) & 813 & 834 & 835 & 841 & 33,7 & $\mathrm{~ns}$ & $\mathrm{~ns}$ & $\mathrm{~ns}$ \\
\hline
\end{tabular}

T- : régime témoin négatif couvrant $73 \%$ des besoins des microbes en $\mathrm{N}$ dégradable ; $\mathrm{U}, \mathrm{UC}$ et $\mathrm{C}$ : régimes supplémentés de manière à couvrir $95 \%$ des besoins des microbes par de l'urée seule (U), un mélange 50:50 d'urée et de caséine (UC) ou de la caséine seule(C). ${ }^{a}$ écart-type résiduel de l'analyse de variance.

$20 \mathrm{mg} / \mathrm{L}$ ont été observées quelques heures par jour (fig 1). Des teneurs en $\mathrm{NH}_{3}$ proches de $50 \mathrm{mg} / \mathrm{L}$ sont généralement reconnues comme nécessaires pour assurer une croissance microbienne optimale et ne pas limiter la digestion des parois (Satter et Slyter, 1974 ; Hoover, 1986). Ainsi la disponibilité en $\mathrm{NH}_{3}$ a pu être un facteur limitant pour assurer une digestion microbienne optimale avec le régime $T$-. La supplémentation par de l'azote dégradable a conduit à des concentrations en $\mathrm{NH}_{3}$ beaucoup plus élevées et probablement non limitantes. Toutefois, la teneur moyenne et le pic postprandial de $\mathrm{NH}_{3}$ ont légèrement diminué avec l'accroissement de l'apport de caséine peut être parce qu'une partie de la caséine n'a pas été dégradée jusqu'au stade $\mathrm{NH}_{3}$ ou que, du fait d'une dégradation plus lente que celle de l'urée, le $\mathrm{NH}_{3}$ produit a été réutilisé par les cellules microbiennes. Une fraction de la caséine a aussi pu échapper à la dégradation ruminale, mais cette hypothèse est peu probable dans la mesure où le flux d' $\mathrm{N}$ d'origine alimentaire n'a pas été accru entre le régime $U$ et les régimes $U C$ et $C$. Ces résultats suggèrent que le taux de captation de l'azote de la caséine, a priori retenu identique à celui de l'urée $(0,8)$, a pu être légèrement sous-estimé. Un taux de captation de 0,9 , identique à celui fixé pour les protéines dégradables dans le système PDI (Vérité et Peyraud, 1989), aurait conduit à utiliser des quantités légèrement plus faibles de caséine ( 2,9 vs $3,2 \%$ MS pour le régime $\mathrm{C}$ ) et ainsi à limiter les effets digestifs observés.

La proportion d'AGVR a augmenté avec la proportion de caséine dans le régime. En admettant une production moyenne de $9 \mathrm{~mol}$ d'AGV par kg de MO réellement fermentée dans le rumen (Sauvant, 1996), l'apport d'AGVR se serait accru de 2,6 à $3,6 \mathrm{~mol} /$ jour entre les régimes sans et avec caséine. Cet accroissement des quantités d'AGVR traduit sans doute aussi celui d'autres chaînes carbonées telles que des acides aminés et de petits peptides qui sont également des facteurs de croissance in vitro de la microflore cellulolytique (Hungate, 1966 ; Maeng et al, 1976 ; Argyle et Baldwin, 1989). Ces données suggèrent donc que la fourniture en nutriments azotés pour les microbes a bien été accrue par l'apport de caséine alors que l'urée n'a permis d'accroître que la disponibilité en $\mathrm{NH}_{3}$.

\section{Digestion de la MO et des constituants pariétaux}

La digestibilité de la MO a été significativement plus élevée pour les trois régimes 
supplémentés en $\mathrm{N}$ dégradable mais la différence est restée assez modérée $(+0,025$ en moyenne). Des résultats similaires avaient déjà été obtenus pour des vaches laitières alimentées avec des régimes à base d'ensilage de maïs (Vérité, 1978 ; fig 2). En fait, l'effet d'une supplémentation en $\mathrm{N}$ dégradable sur la digestibilité est d'autant plus important que le régime est, à l'origine, pauvre en MAT. Ainsi, avec des moutons alimentés avec de l'ensilage de maïs, l'apport d'une même dose d'urée accroît la digestibilé de 0,03 unité lorsque la teneur en MAT de l'ensilage est comprise entre 7 et $10 \%$ mais de 0,06 unité lorsque l'ensilage contient moins de $6 \%$ de MAT (Andrieu et Demarquilly, 1974). De même, sur bouvillons alimentés avec des régimes à base de foin, Kropp et al (1977) ont mis en évidence un accroissement de la digestibilité de 0,52 à 0,56 puis 0,58 lorsque la teneur en MAT du régime a été accrue de 8 à 11 , puis $13 \%$. De même, Strokes et al (1988) ont noté un accroissement de la digestibilité de 0,58 à 0,67 , puis 0,69 pour des teneurs en MAT de 6, 9 et $11 \%$ respectivement. Cette évolution de la digestibilité de la MO a été liée à celle des constituants pariétaux, notamment au niveau du rumen (tableau III). D'autres études avaient déjà mis en évidence une augmentation importante de la digestibilité ruminale des parois en relation avec un apport d' $\mathrm{N}$ dégradable (Kropp et al, 1977 ; Strokes et al, 1988). Cet accroissement de la digestibilité des parois peut être relié à une plus grande disponibilité en

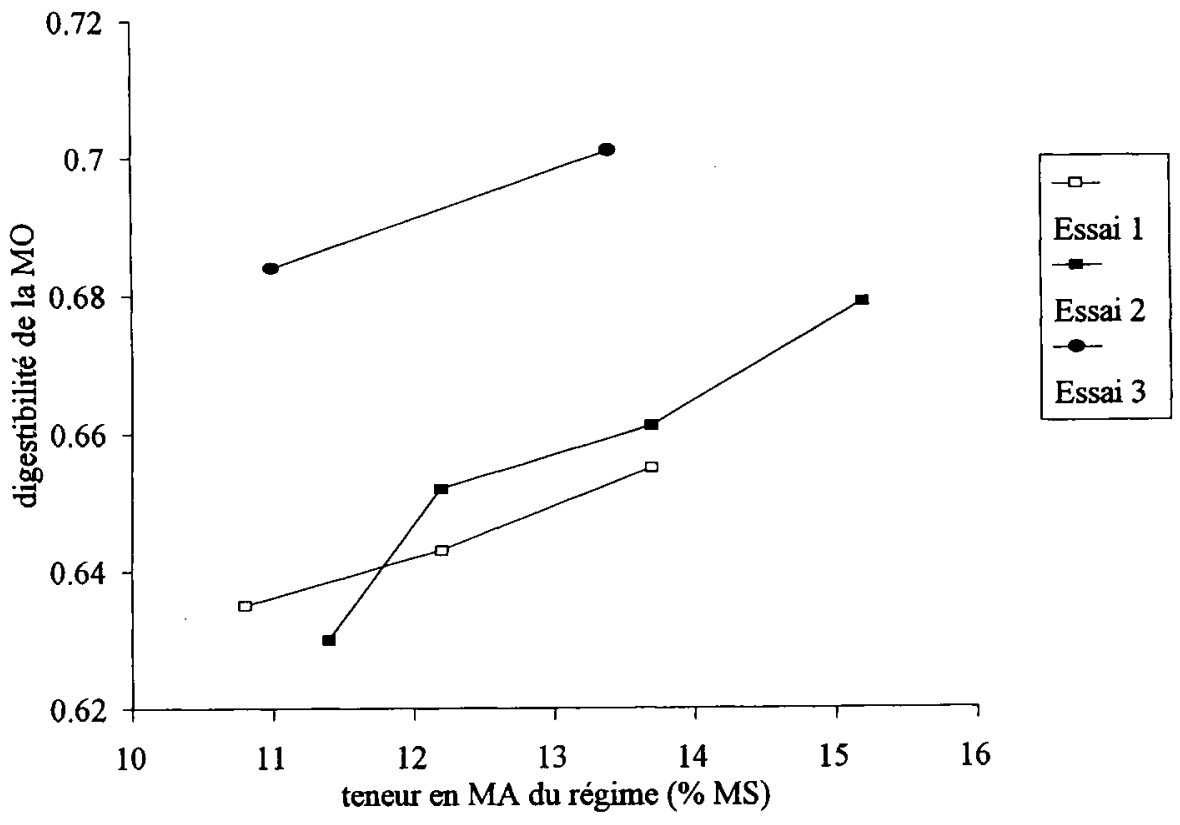

Fig 2. Effet du niveau d'apport de matières azotées dégradables sur la digestibilité de la matière organique des régimes à base d'ensilage de maïs chez la vache laitière. L'accroissement du niveau d'apport a été obtenu par addition de doses croissantes d'urée. Essai 1 et 2 (Vérité, 1978), Essai 3 (présent essai). 
$\mathrm{NH}_{3}$ pour les microbes. Ainsi, Kennedy et al (1992) ont mis en évidence une réduction de la vitesse de dégradation des parois de fourrages lorsque la teneur en $\mathrm{NH}_{3}$ du rumen est inférieure à $50 \mathrm{mg} / \mathrm{L}$.

Les digestibilités totales et ruminales de la MO et des constitutants pariétaux ont augmenté avec l'apport de caséine dans la ration. La substitution iso-azotée de l'urée par des sources d'azote dégradable telles que la caséine, le tourteau de soja ou de tournesol se traduit en général par un accroissement modéré (de 0,01 à 0,03 unité) de la digestibilité dans l'ensemble du tube digestif et dans le rumen de la MO et des parois (Amos et Evans, 1976 ; Stock et al, 1986 ; Cecava et al, 1988). A l'inverse, l'introduction de protéines peu dégradables en substitution de l'urée s'accompagne d'une réduction de la digestion ruminale de la $\mathrm{MO}$ (Cottrill et al, 1982 ; Stock et al, 1986 ; Cecava et Parker, 1993). Les quantités de MO et de parois fermentées ont été maximales avec les régimes contenant de la caséine malgré des teneurs en $\mathrm{NH}_{3}$ un peu plus faibles que pour le régime $\mathrm{U}$. Il apparaît donc que la faible teneur en $\mathrm{NH}_{3}$ n'était pas le seul facteur limitant de l'activité cellulolytique avec le régime $\mathrm{T}$ - et qu'une partie de l'effet peut sans doute s'expliquer par l'apport d'AGVR et/ou d'acides aminés et de peptides avec la caséine. Cependant, la réponse est restée faible au regard de l'écart de disponibilité en AGVR. Il est donc probable que les rations $T$ - et $U$ couvraient déjà l'essentiel des besoins des microbes en chaînes carbonées. Cette faible réponse pourrait aussi être liée à une dégradation rapide de la caséine qui a pu limiter la disponibilité en chaînes aminées dans le temps. En effet, Stritzler et al (1992) avec une étude in sacco et Merry et al (1990) en fermenteur en continu ont mis en évidence un accroissement de l'activité cellulolytique plus important lors d'une supplémentation avec de la farine de poisson qu'avec de la caséine ou du tourteau de soja.
La digestibilité de l'amidon n'a que peu été affectée par le niveau et la nature de l'azote dégradable. Ce résultat est en accord avec les données de Veira et al (1980) et Schaefer et al (1980). Ces derniers auteurs ont montré que seules des teneurs en $\mathrm{NH}_{3}$ dans le rumen inférieures à $20 \mathrm{mg} / \mathrm{L}$ limitent la croissance de Bacterö̈des amylophilus, une des principales espèces bactériennes fermentant l'amidon. Ce résultat est aussi en accord avec les données de Thomson et Johnson (1984) qui avaient démontré que la présence de chaînes aminées préformées était moins importante pour les bactéries utilisant l'amidon que pour celles utilisant la cellulose.

La réduction de 0,02 à 0,03 unités de la digestibilité de la MO lors d'une réduction importante des apports d'azote dégradable correspond à une réduction de la teneur en énergie nette du régime de 0,05 UFL environ (Vérité, 1978), ce qui est cohérent avec la réduction de la production laitière observée entre les traitements $T$ - et ceux contenant de la caséine.

\section{Digestion de l'azote}

Le flux d'azote entrant dans l'intestin et l'efficacité des synthèses microbiennes n'ont pas été affectés par l'accroissement du niveau d'apport d'azote dégradable. $\mathrm{Ce}$ résultat est en bon accord avec des travaux antérieurs (Kropp et al, 1977 ; Redman et al, 1980 ; Veira et al, 1980). L'absence d'effet de la supplémentation du régime Tpar de l'urée suggère que des niveaux faibles d'ammoniac dans le rumen ne constituent pas per se le principal facteur limitant les synthèses microbiennes. Dans leur étude, Veira et al (1980) n'ont d'ailleurs pas observé de corrélation entre l'efficacité des synthèses microbiennes et les teneurs en $\mathrm{NH}_{3}$ du rumen qui variaient de 19 à $130 \mathrm{mg} / \mathrm{L}$. A partir d'une revue de la littérature sur vaches laitières, Clark et al (1992) ont avancé qu'une valeur seuil de $25 \mathrm{mg}$ de 
$\mathrm{NH}_{3}$ serait suffisante pour maximiser la protéosynthèse in vivo. Avec le régime T-, la concentration en $\mathrm{NH}_{3}$ a été sensiblement plus élevée et n'a atteint des valeurs proches de $25 \mathrm{mg} / \mathrm{L}$ que quelques heures par jour (fig 1).

La supplémentation par de la caséine n'a entraîné aucun accroissement de l'efficacité des synthèses microbiennes et du flux d'azote microbien. La fourniture d'AGVR et/ou de chaînes aminées n'a donc pas été un facteur limitant de la protéosynthèse. Le flux d'azote microbien et l'efficacité des synthèses ne sont généralement pas affectés (Chen et al, 1992 ; Cecava et Parker, 1993) ou affectés de manière très modérée (Amos et Evans, 1976 ; Cecava et al, 1988) par la nature de la source d'azote dégradable lorsque l'urée représente moins de $30 \%$ de l'azote total du régime, ce qui a été le cas dans notre étude. En fait, le remplacement de l'N uréique par de la caséine n'accroît l'efficacité des synthèses que dans le cas de régimes synthétiques ne contenant pratiquement pas de protéines (Hume, 1970 ; Leibholz et Kellaway, 1979). Ainsi, Stock et al (1986) ont observé un accroissement important du flux de NNA et de la protéosynthèse en remplaçant $5 \%$ de l'urée par de la caséine lorsque l'urée représentait $95 \%$ de l'azote dégradable du régime témoin, mais aucun effet de cette substitution lorsque l'urée ne représentait plus que 20 ou même jusqu'à $80 \%$ de l'azote dégradable des régimes.

Ainsi, les besoins spécifiques en $\mathrm{NH}_{3}$ et en chaînes carbonées préformées semblent correctement couverts pour assurer une protéosynthèse efficace dans les régimes à base d'ensilage de maïs. Les effets réels d'une complémentation par des sources de protéine dégradable en substitution de l'urée sont alors très ténus et à la limite de ce qui peut être décelé par les techniques d'étude de la digestion. Ce résultat est en bonne cohérence avec les essais de production (Vérité, non publié) mettant en évidence l'absence d'effet net de la source de $\mathrm{N}$ dégradable sur la production de lait et de protéines chez des vaches alimentées avec des régimes à base d'ensilage de maïs. En revanche, les besoins des bactéries cellulolytiques sont plus difficiles à couvrir et nos données indiquent qu'il est prudent de ne pas apporter plus de $20 \%$ de l'azote dégradable par de l'urée si l'on veut maximiser la digestibilité ruminale des parois. Hoover (1986) avait déjà rapporté de nombreuses études in vitro dans lesquelles la croissance microbienne était maximisée à des niveaux d'ammoniac plus faibles que ceux nécessaires pour maximiser la cellulolyse. Les besoins spécifiques des bactéries impliquées dans la dégradation des parois semblent donc un peu plus difficiles à couvrir, peut-être parce que ces bactéries sont fixées aux particules alimentaires et, de ce fait, sont exposées à un environnement moins riche en $\mathrm{NH}_{3}$ et en chaînes carbonées que le liquide ruminal (Hoover, 1986).

\section{Réduction des apports azotés et métabolisme de l'azote}

Malgré une quantité d' $N$ ingéré beaucoup plus faible, le flux de NNA à l'entrée de l'intestin n'a pas été différent entre le régime T- et les trois régimes supplémentés. En outre, la nutrition azotée de l'animal ne semble pas avoir été affectée puisque la quantité de protéines sécrétées dans le lait n'a pas été sensiblement modifiée. Ces données mettent en évidence les possibilités de recyclage chez la vache laitière et confirment d'autres données obtenues avec des régimes de fourrages verts par Peyraud et al (1997). Ce résultat s'explique par un gain net important d'azote entre la ration et le duodénum. Les quantités nettes d'azote recyclé ont été beaucoup plus élevées avec le régime $T$ - que pour les régimes supplémentés (110 vs $31 \mathrm{~g} /$ jour). Pour un état de nutrition azotée des animaux très semblable ( $\mathrm{N}$ exporté dans le lait représente $30 \%$ du 
flux de NNA pour les quatre traitements), cette différence au niveau de $\mathrm{N}$ recyclé est à rapprocher de l'épargne d'azote uréique au niveau de l'urine ( 22 vs 80 g/jour respectivement pour les régimes $\mathrm{T}$ - et supplémentés). Pour le régime T-, les quantités d'azote uréique exportées dans l'urine et l'urémie ont été très faibles. Ceci indique que les limites maximales d'adaptation des animaux producteurs à des régimes pauvres en $\mathrm{N}$ ont quasiment été atteintes. La nature de l'azote dégradable n'a pas affecté les quantités d'azote sous forme d'urée urinaire. Ce résultat est à relier au bilan quantitatif de la digestion ruminale de $\mathrm{N}$ qui n'a pas été affecté malgré des cinétiques de production de $\mathrm{NH}_{3}$ un peu plus marquées avec les régimes contenant de l'urée.

\section{Niveau d'ingestion des animaux}

Il faut rappeler que les résultats ont été obtenus avec des quantités ingérées légèrement limitées. En situation d'alimentation à volonté, l'ingestion volontaire de MS peut être fortement affectée par le niveau d'apport d'azote dégradable. Ainsi, chez la vache laitière, l'ingestion de fourrage distribué à volonté s'accroît avec la teneur en MAT du régime jusqu'à des teneurs proches de $14 \%$ (Huber et al, 1976 ; Journet et Rémond, 1976). De même, de nombreuses études conduites sur des bovins alimentés avec des foins pauvres en MAT $(<9-10 \%)$ ont mis en évidence un accroissement des quantités de MS ingérées de 10 à $30 \%$ lors d'une supplémentation avec des protéines dégradables (Mc Collum et Galyean, 1985 ; Strokes et al, 1988). Cette amélioration de l'ingestion peut s'expliquer par l'amélioration de l'activité microbienne et, en conséquence, de la vitesse de digestion des parois ainsi que par l'amélioration de l'état de nutrition azotée des animaux (Kempton et al, 1977). La transposition des données obtenues dans cet essai au cas d'animaux alimentés à volonté doit donc être effectuée avec prudence. Il n'est en particulier pas souhaitable de recommander une réduction des apports d'N dégradable aussi importante que celle utilisée pour le régime $\mathrm{T}$ De même, un effet légèrement positif sur l'ingestion de fourrage d'une supplémentation par des protéines dégradables, en substitution de l'urée, ne peut être totalement exclu en situation d'alimentation à volonté.

\section{CONCLUSION}

En alimentation à $95 \%$ de l'ad libitum, une réduction importante des apports d'azote dégradable entraîne une réduction modérée de la digestibilité de la ration mais n'affecte pas sa valeur azotée malgré un métabolisme de l'azote très différent, lequel conduit à une limitation importante des rejets azotés. Dans un contexte général de réduction des rejets azotés par les troupeaux laitiers, les résultats de cet essai laissent donc envisager qu'il est possible de tolérer des déficits en azote dégradable un peu plus élevés que ceux admis actuellement (Vérité et Peyraud, 1989). La nature de l'azote dégradable a peu d'effet sur la nutrition des vaches laitières car les besoins spécifiques en chaînes carbonées des microorganismes semblent être couverts dans la majorité des rations classiquement utilisées. Afin de ne pas limiter la digestion des parois, il semble toutefois nécessaire de veiller à ne pas dépasser $20 \%$ d'N uréique dans l'azote dégradable total du régime.

\section{REMERCIEMENTS}

La présente étude a été réalisée avec le financement du Groupement Coopératif de Recherche sur la vache laitière (Ucanor, Ccpa, Unicopa) et des fonds du Sypram. Les auteurs tiennent aussi à remercier $\mathrm{M}$ Hétault et son équipe pour l'entretien des animaux et toute l'équipe du laboratoire Inra de St Gilles pour les analyses réalisées. 


\section{RÉFÉRENCES}

Amos HE, Evans J (1976) Supplementary protein for low quality bermudagrass diets and microbial protein synthesis. J Anim Sci 43, 861-868

Andrieu J, Demarquilly C (1974) Valeur alimentaire du maïs fourrage. II. Influence du stade de végétation, de la variété, du peuplement, de l'enrichissement en épis et de l'addition d'urée sur la digestibilité et l'ingestibilité de l'ensilage de maïs. Ann Zootech 23, $1-25$

Argyle JL, Baldwin RL (1989) Effects of amino acids and peptides on rumen microbial growth yields. $J$ Dairy Sci 72, 2017-2027

Cecava MJ, Parker JE (1993) Intestinal supply of amino acids in steers fed ruminally degradable and undegradable crude protein sources alone and in combination. J Anim Sci 71, 1596-1605

Cecava MJ, Merchen NR, Berger LL, Fahey GC Jr (1988) Effects of dietary energy level and protein source on site of digestion and duodenal nitrogen and amino acid flows in steers. J Anim Sci 66, 961974

Chen XB, GU CX, Zhang WX, Orskov ER (1992) Rumen microbial protein supply to sheep given diets containing either urea or casein as the main $\mathbf{N}$ source. Br Soc Anim Pro, paper n 215

Clark JH, Klusmeyer TH, Cameron MR (1992) Symposium: nitrogen metabolism and amino acid nutrition in dairy cattle. $J$ Dairy $S c i$ 75, 2304-2323

Cotta MA, Russel JB (1982) Effect of peptides and amino acids on efficiency of rumen bacterial protein synthesis in continuous culture. J Dairy $S_{c i} 65$, 226-234

Cottrill BR, Beever DE, Austin AR, Osbourn DF (1982) The effect of protein- and non-protein-nitrogen supplements to maize silage on total amino acid supply in young cattle. $B r J$ Nutr 48, 527-541

Giger S, Pochet S (1987) Méthodes d'estimation des constituants pariétaux dans les aliments destinés aux ruminants. Bull Tech CRZV Theix, Inra 70, 4960

Hoover WH (1986) Chemical factors involved in ruminal fiber digestion. J Dairy Sci 69, 2755-2766

Huber JT, Boman RL, Henderson HE (1976) Fermented ammoniated condensed whey as a nitrogen supplement for lactating cows. I Dairy Sci 59, 19361943

Hume ID (1970) Synthesis of microbial protein in the rumen. III. The effect of dietary protein. Aust $J$ Agric Res 21, 305-314

Hungate RE (1966) The rumen and its microbes. Academic Press, Inc, New York, USA

Hyden S (1955) A turbidimetric method for turbidimetric determination of higher polyethylene glycols in biological materials. Ann R Agric Col Sweden $21,139-145$
Jouany JP (1982) Volatile fatty acid and alcohol determination in digestive contents, silage juices, bacterial cultures and anaerobic fermentor contents. Sci aliments 2, 131-144

Journet M, Rémond B (1976) Physiological factors affecting the voluntary intake of feed by cows: a review. Livest Prod Sci 3, 129-146

Kempton TJ, Nolan JV, Leng RA (1977) Principles for the use of nonprotein nitrogen and by-pass protein in diets for ruminants. World Anim Rev 22, 2 19

Kennedy PM, Boniface AN, Liang ZJ, Muller D, Murray $R M$ (1992) Intake and digestion in swamp buffaloes and cattle. 2 . The comparative response to urea supplements in animals fed tropical grasses. $J$ Agric Sci Camb 119, 243-254

Kropp JR, Johnson RR, Males JR, Owens FN (1977) Microbial protein synthesis with low quality roughage rations: level and source of nitrogen. J Anim Sci 46, 844-854

Le Hénaff L (1991) Importance des acides aminés dans la nutrition des vaches laitières. Thèse, université Rennes I, France

Leibholz J, Kellaway RC (1979) Amino acid requirements for microbial protein synthesis, Ann Rech Vet 10, 274-276

Maeng WJ, Van Nevel CJ, Baldwin RL, Morris JG (1976) Rumen microbial growth rates and yields: effects of amino acids and protein. J Anim Sci 59, 68-79

Malawer SJ, Powell DW (1967) An improved turbidimetric analysis of polyethylene glycol utilizing an emulsifier. Gastroent 53, 250-256

Mambrini M, Peyraud JL (1994) Mean retention time in digestive tract and digestion of fresh perennial ryegrass by lactating dairy cows: influence of grass maturity and comparison with a maize silage diet. Reprod Nutr Develop 34, 9-23

Mc Collum FT, Galyean ML (1985) Influence of cottonseed meal supplementation on voluntary intake, rumen fermentation and rate of passage of prairie hay in beef steers. J Anim Sci 60, 570-577

Merry RJ, Mac Allan AB, Smith RJ (1990) In vitro continuous culture studies on the effect of nitrogen source on rumen microbial growth and fiber digestion. Anim Feed Sci Technol 30, 55-64

Michel MC (1971) Analyse quantitative de quelques substances azotées et glucidiques en milieu biologique. Essai de rationalisation. Thèse de doctorat, université de Clermont-Ferrand, France

Peyraud JL, Astigarraga L, Faverdin P (1997) Digestion of fresh perennial ryegrass fertilized at two levels of nitrogen by lactating dairy cows. Anim Feed Sci Technol (sous presse)

Pittman KA, Bryant MP (1964) Peptides and others nitrogen sources for growth of Bacteroïdes ruminicola. I Bacteriol $88,401-410$ 
Redman RG, Kellaway RC, Leibholz J (1980) Utilization of low quality roughages: effects of urea and protein supplements of differing solubility on digesta flows, intake and growth rate of cattle eating oaten chaff. Br J Nutr 44, 343-354

SAS (1987) SAS User's Guide, statistics SAS Institute, Carry, NC, USA

Satter LD, Slyter LL (1974) Effect of ammonia concentration on rumen microbial protein production in vitro. Brit J Nutr 32, 199-208

Sauvant D (1996) A comparative evaluation of models of lactating ruminant. Ann Zootech 45, suppl, 215235

Schaefer DM, Davis CL, Bryant MP (1980) Ammonia saturation constants for predominant species of rumen bacteria. J Dairy Sci 63, 1248-1263

Stock R, Klopfenstein T, Brink D, Britton R, Harmon $D$ (1986) Whey as a source of rumen-degradable protein. I. Effects on microbial protein production. J Anim Sci 63, 1561-1573

Stritzler NP, Wolstrup J, Eggum BO, Jensen BB (1992) Factors affecting degradation of barley straw in sacco and microbial activity in the rumen of cows fed fibre-rich diets. 1. The source of supplemental nitrogen. Anim Feed Sci Tech 38, 263-280

Strokes SR, Goetsch AL, Jones AL, Landis KM (1988) Feed intake and digestion by beef cows fed prai- rie hay with different levels of soyabean meal and receiving postruminal administration of antibiotics. J Anim Sci 66, 1778-1789

Thomson KV, Johnson F (1984) Availability of nitrogen in different protein sources, particularly as regards to fish viscera silage for in vitro digestibility of starch and cellulose. Acta Agric Scand 34, 1725

Van Soest PJ (1963) Use of detergent analysis of fibrous feed. II. A rapid method for the determination of fiber and lignin. $J$ Assoc Off Chem 46,829 . 835

Veira DM, Mac Leod GK, Burton JH, Stone JB (1980) Nutrition of the weaned holstein calf. I. Effect of dietary protein level on rumen metabolism. J Anim Sci 50, 937-944

Vérité R (1978) Rationnement azoté. In : La vache Laitière, 183-197. Inra Publications, 78000 Versailles, France

Vérité R, Peyraud JL (1989) Protein: the PDI system. In: Ruminant Nutrition: recommanded allowances and feed tables ( $\mathrm{R}$ Jarrige ed) John Libbey, Inra, London, UK ; Paris, France, 33-48

Wright DE (1967) Metabolism of peptides by rumen microorganisms. Appl Microbiol 15, 547-550 